\title{
Simulación de la eficiencia de la utilización de nitrógeno en novillos finalizados a pastoreo en el sur de Chile
}

\author{
Simulation of the nitrogen use efficiency in pasture-finished steers in southern Chile \\ RA Arias ${ }^{\text {ab }}$, A Velásquez ${ }^{\mathrm{ab}}, \mathrm{M}$ Toneatti ${ }^{\mathrm{a}}$ \\ ${ }^{a}$ Escuela de Agronomía, Facultad de Recursos Naturales Universidad Católica de Temuco, Temuco, Chile.

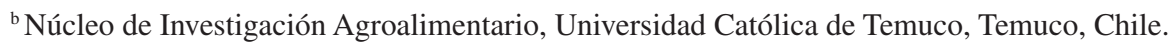

\begin{abstract}
SUMMARY
The high content of crude protein $(\mathrm{CP})$ of pastures in southern Chile creates a nutritional imbalance that results in large amount of nitrogen $(\mathrm{N})$ being excreted into the environment. Two regression equations and a dynamic model, available from the literature, were utilized to simulate the $\mathrm{N}$ use efficiency (NUE) in pasture-finished steers. A medium frame animal (Angus breed) with an initial and final body weight (BW) of 350 and $500 \mathrm{~kg}$, respectively, was used to estimate nutrient requirements and excretions. Average DMI was fixed at 2.2\% of animal BW with 150 days on feed (October to February). Energy and protein requirements, as well as the retained protein, were estimated by using Beef NRC software (tabular system level 1), while the nutritional characteristics of the pasture were obtained from studies based in Chile. The quality of the pastures was good enough to allow daily weight gain (GDP) of $1.0 \mathrm{~kg} / \mathrm{d}$. However, metabolizable protein balance was positive across the whole finishing period (average of $149 \mathrm{~g} / \mathrm{d}$ ). Total production of microbial protein increases with time because of the greater DMI, even when the PC content in forage decreases with time. This also explains the lower contribution of UIP during the last months of the finishing period. The estimated $\mathrm{N}$ intake was $123 \mathrm{~g} / \mathrm{d}$, whereas $\mathrm{N}$ excretion was 106 $\mathrm{g} / \mathrm{d}$. In summary, the combination of high levels of CP of Chilean southern pastures and the low NUE of the finishing cattle (13.4 - 16.3\%), represent a strong challenge in terms of contamination and production.
\end{abstract}

Key words: nitrogen excretion, metabolizable protein system, pasture finishing system.

\section{RESUMEN}

El alto contenido de proteína cruda de las praderas del sur de Chile provoca un desbalance nutricional que genera una gran cantidad de nitrógeno (N) excretado al medioambiente. Dos ecuaciones de regresión y un modelo dinámico, disponibles en la literatura, fueron utilizadas para simular la eficiencia de uso de nitrógeno (EUN) en una engorda de novillos finalizados a pastoreo. Para efectos de cálculo se consideró un novillo de estructura corporal media (raza Angus), peso vivo inicial de $350 \mathrm{~kg}$ y final de $500 \mathrm{~kg}$. El consumo promedio de materia seca (MS) se fijó en 2,2\% del peso vivo y el periodo de engorda en 150 días (octubre a febrero). Los requerimientos de energía y proteína, así como de proteína retenida, fueron estimados mediante el software del NRC de carne (sistema tabular, nivel 1), mientras que las características nutricionales de la pradera fueron obtenidas de publicaciones nacionales. Los resultados de la simulación indican que la calidad de la pradera es lo suficientemente buena para permitir ganancia diaria de peso de $1,0 \mathrm{~kg} / \mathrm{d}$. Sin embargo, el balance de proteína metabolizable fue positivo durante todo el período de engorda, promediando 149 g/d. La producción total de proteína microbiana aumenta con el tiempo debido al mayor consumo de MS, aún cuando el contenido del proteína cruda en el forraje disminuye, lo que también explica la menor contribución de proteína no degradable en el rumen en los últimos meses. La estimación $\mathrm{N}$ consumido fue de $123 \mathrm{~g} / \mathrm{d}$, mientras que la excreción alcanzó $106 \mathrm{~g} / \mathrm{d}$. En conclusión, la combinación de altos niveles de proteína cruda de las praderas del sur de Chile y la baja EUN del ganado (13,4 a 16,3\%), representan un fuerte desafío en términos de contaminación y producción.

Palabras clave: nitrógeno excreción, proteína metabolizable, engorda a pastoreo.

\section{INTRODUCCIÓN}

El nitrógeno $(\mathrm{N})$ constituye un importante elemento en la producción animal porque forma parte de los aminoácidos que constituyen la proteína de tejidos, leche, huevos y lana (Elizondo 2002, Francis y col 2007, Van der Hoek 1998). Además, constituye un factor relevante en la producción de forraje, el que a su vez proporciona

Aceptado: 29.11.2012.

* Escuela de Agronomía Casilla 15-D, Temuco, Chile; rarias@uct.cl más del 70\% de la ingesta de proteína en los animales (Van der Hoek 1998). El N es el elemento más abundante entre los gases que constituyen la atmósfera (78\%), encontrándose en su forma molecular dinitrógeno $\left(\mathrm{N}_{2}\right)$ y trazas de $\mathrm{N}_{2} \mathrm{O}$ (Capone y col 2006). Sin embargo, las plantas no pueden utilizarlo directamente en esta forma, ya que las raíces lo absorben en forma de iones nitrato $\left(\mathrm{NO}_{3}^{-}\right)$y amonio $\left(\mathrm{NH}_{4}^{+}\right)$luego de que microorganismos del suelo fijan y transforman el $\mathrm{N}_{2}$ (Oaks 1994). Es también el nutriente con mayor impacto sobre el rendimiento y la calidad de los cultivos agrícolas y es extremadamente dinámico en el suelo. Desafortunadamente, la produc- 
ción de cultivos y en particular la producción animal, aprovechan este recurso con una tasa de eficiencia muy baja (Steinfield y col 2006). En efecto, las actividades agrícolas y la utilización de combustibles fósiles han causado un incremento en las emisiones globales de nitrógeno reactivo $(\mathrm{Nr})^{1}$ en los últimos 50 años (Galloway y col 2004 , Galloway y col 2008). Sin embargo, un exceso de $\mathrm{N}$ en la dieta del animal se traduce en un mayor riesgo de contaminación del medio ambiente, junto a un efecto detrimental en el bienestar animal y en mayores pérdidas económicas para el agricultor (Dumont y Salazar 2001). Las heces y orina eliminados por los animales con un alto contenido de $\mathrm{N}$ pueden causar impactos negativos en la calidad del agua, emisión de olores, servir como sustrato orgánico para el crecimiento de algunos artrópodos y microorganismos, también contribuye a la emisión de compuestos nitrogenados tales como $\mathrm{N}_{2} \mathrm{O}_{\mathrm{NH}_{4}+} \mathrm{y} \mathrm{NO}_{3}^{-}$hacia la atmósfera o aguas subterráneas (Galloway y col 2004, Van Horn y col 1996).

En 1991, la Unión Europea presentó las normas sobre nitratos, las cuales tienen por objeto prevenir la contaminación de las aguas subterráneas y superficiales por este compuesto nitrogenado procedente de fuentes agrícolas. Estas normas establecen medidas obligatorias que implican un límite en la cantidad de estiércol (orina y heces) que se pueden aplicar por unidad de superficie cada año. Este límite tiene consecuencias importantes en la determinación de la carga animal en las explotaciones ganaderas. Por lo tanto, existe un creciente interés en el desarrollo de métodos para predecir la excreción de $\mathrm{N}$ y de medidas de mitigación para reducir el contenido de $\mathrm{N}$ en las heces y la orina en la producción animal (Yan y col 2007).

A nivel global, existen diversos esfuerzos por desarrollar estrategias de alimentación que permitan mejorar la utilización de $\mathrm{N}$ por los animales, disminuyendo las pérdidas y mitigando los impactos ambientales asociados a estas explotaciones. Una reducción del exceso de $\mathrm{N}$ en la dieta disminuye el contenido de $\mathrm{N}$ en las heces y orina, por lo que la elaboración de dietas que se ajusten a los requerimientos de los animales ayuda a prevenir la excesiva excreción de N y otros nutrientes (Galyean 2000, Klopfenstein y col 2002). En la zona centro-sur de Chile, la combinación de condiciones edafoclimáticas y del ma-

El término nitrógeno reactivo ( $\mathrm{Nr}$ ) incluye todos los compuestos $\mathrm{N}$ en la atmósfera y la biosfera de la Tierra biológicamente activos, fotoquímicamente reactivos, y radiativamente activos. Así $\mathrm{Nr}$ incluye formas reducidas inorgánicas de $\mathrm{N}$ (e.g. $\mathrm{NH}_{3}, \mathrm{NH}_{4}^{+}$), las formas oxidadas inorgánicas (e.g, NOx, $\mathrm{HNO}_{3}, \mathrm{~N}_{2} \mathrm{O}, \mathrm{NO}_{3}$ ), y compuestos orgánicos (urea, aminas, proteínas, ácidos nucleicos). Esta definición es mucho más amplia que el término "Nitrógeno reactivo" definido por la comunidad química (Galloway JN, FJ Dentener, DG Capone, EW Boyer, RW Howarth, SP Seitzinger, GP Asner, CC Cleveland, PA Green, EA Holland, DM Karl, AF Michaels, JH Porter, AR Townsend, CJ Vorosmarty. 2004 Nitrogen cycles: past, present, and future. Biogeochemistry 70, 153-226. nejo de las praderas, permite una gran producción de materia seca con muy buenas características nutricionales. Sin embargo, en muchas ocasiones el contenido de proteína cruda (PC) puede alcanzar valores cercanos o por sobre el 30\% (Balocchi 2011, Pulido y col 2010). Estos valores son muy superiores a los que necesita un animal en engorda o bien una vaca de alta producción, generando un problema de desbalance nutricional y una consecuente contaminación ambiental por N (Keim y Anrique 2011).

El objetivo del presente trabajo fue determinar, mediante un modelo de simulación, la eficiencia del uso del $\mathrm{N}$ en sistemas de engorda de novillos en base a praderas del sur de Chile, estableciendo una línea de base que permita desarrollar futuras investigaciones y evaluar estrategias de mitigación de impacto ambiental en esta área.

\section{MATERIAL Y MÉTODOS}

El presente estudio corresponde a una simulación que utilizó como información base los trabajos realizados por Guo y Zoccarato (2005) y Yan y col (2007). El primer trabajo corresponde al desarrollo de un modelo dinámico para simular la excreción de $\mathrm{N}$ en ganado en engorda en forma simple e intuitiva. El principio del modelo señala que la excreción de $\mathrm{N}$ es equivalente a la diferencia entre $\mathrm{N}$ consumido menos el $\mathrm{N}$ retenido en el cuerpo. Este último puede ser fácilmente estimado como la diferencia entre el contenido de $\mathrm{N}$ del animal al inicio y final del periodo de engorda. El modelo de Guo y Zoccarato utiliza como información base: el peso inicial del animal, la ganancia de peso diario esperada y el consumo de $\mathrm{N}$. Por su parte, Yan y col (2007) establecieron una serie de ecuaciones para predecir la cantidad de $\mathrm{N}$ excretado por el ganado bovino de carne. En su investigación, Yan y col (2007) utilizaron datos de 286 animales de distintas edades, razas (Holstein, Friesian, Aberdeen Angus, Simmental, Charolais, Limousin, Belgian Blue y Blonde D'Aquitaine) y pesos vivos (153 a $580 \mathrm{~kg}$ ). Los datos se obtuvieron de 14 experimentos conducidos entre 1984 y 2003 en el norte de Irlanda. Las dietas ofrecidas incluyeron entre un 20 y $100 \%$ de forraje, cuyo contenido de $\mathrm{PC}$ varió entre 10 y $22 \%$ base materia seca; mientras que los rangos de $\mathrm{N}$ consumido y excretado correspondieron a: 73-316 y 43-227 g/d, respectivamente. Para esta simulación, se seleccionaron dos de las siete ecuaciones obtenidas por Yan y col (2007), utilizando como criterio de selección el valor de su coeficiente de determinación $\left(\mathrm{R}^{2}\right)$, su simplicidad y tipo de información requerida. Lo anterior resultó en la selección de las ecuaciones 1 y 2 que se presentan a continuación:

Excreción N, $(\mathrm{g} / \mathrm{d})=[0,550+0,032 \times \mathrm{CN} / \mathrm{CMS}] \mathrm{x}$ $\mathrm{PV}^{0,75}+15$

Excreción $\mathrm{N},(\mathrm{g} / \mathrm{d})=0,609+\mathrm{CN}+0,599 \mathrm{x}$ $\mathrm{PV}^{0,75}-24,7$ 
Donde: $\mathrm{CN}=$ consumo de nitrógeno $(\mathrm{g} / \mathrm{d}) ; \mathrm{CMS}=$ consumo de materia seca $(\mathrm{kg} / \mathrm{d})$; y PV = peso vivo del animal $(\mathrm{kg})$. Para el cálculo de los requerimientos nutricionales y de excreción de $\mathrm{N}$ se consideró un animal de raza Angus de estructura corporal media (Frame score 6), cuyas principales características se detallan en el cuadro 1. El consumo promedio de materia seca para la engorda se fijó en un 2,2\% del peso vivo del animal. El periodo de engorda consideró un total de 150 días, comprendido entre los meses de octubre a febrero. Los requerimientos de energía y proteína, así como de proteína retenida por el animal fueron estimados mediante el software NRC para carne, utilizando el sistema tabular nivel 1 (NRC 2000) cuyos resultados se presentan en el cuadro 2 .

Cuadro 1. Características generales del novillo utilizado para estimar excreción y eficiencia de nitrógeno.

General characteristics of the steer used to estimate nitrogen excretion and efficiency.

\begin{tabular}{lc}
\hline Peso inicial, kg & 350 \\
Peso final, kg & 500 \\
Peso promedio, kg & 425 \\
Ganancia peso diaria minima, kg/d & 1,00 \\
Peso estimado para lograr un bajo nivel de & 520 \\
marmoleo kg* & \\
Días en engorda & 150 \\
Edad (meses) & 14,0 \\
Raza & Angus \\
\hline
\end{tabular}

* Estimado según valor del NRC de carne, 2000.
Los requerimientos nutricionales mensuales se estimaron sobre la base del peso vivo promedio para el mes en cuestión con una ganancia de $1,0 \mathrm{~kg} / \mathrm{d}$. Para el cálculo de los requerimientos se consideró una condición termoneutral $\left(25^{\circ} \mathrm{C}\right)$, ausencia de barro y la utilización de implantes anabólicos. Además, se estimó la cantidad de proteína consumida no degradable en el rumen (UIP), y la proteína de origen microbiano mediante las ecuaciones 3 y 4 . Los valores de nutrientes digestibles totales (TDN,) fueron estimados utilizando la ecuación de Weiss y col (1992), mientras que para la degradabilidad de la PC se utilizaron los valores reportados por Anrique y col (2010).

Proteína UIP $(\mathrm{g} / \mathrm{d})=$ CMS $\times$ PC x UIPp x EFa x 1.000 $(\mathrm{g} / \mathrm{kg})$

Proteína microbiana $(\mathrm{g} / \mathrm{d})=\mathrm{CMS} \times \mathrm{TDN}$ x EFm $\mathrm{x}$ $\mathrm{EFa} \times 1.000(\mathrm{~g} / \mathrm{kg})$

En donde: $\mathrm{CMS}=$ consumo de materia seca $(\mathrm{kg} / \mathrm{d})$; $\mathrm{PC}=$ proteína cruda (\%); UIPp = porcentaje de la PC que escapa a la digestión ruminal; $\mathrm{EFa}=$ eficiencia de absorción de proteína microbiana en el intestino delgado del animal, estimado en 64\% según NRC (NRC 2000); TDN $=$ nutrientes digestibles totales $(\%), \mathrm{EFm}=$ eficiencia de microorganismos ruminales en la síntesis de proteína, estimado en 13\% según NRC de carne (NRC 2000). Asimismo, con el objeto de determinar si la pradera suministraba suficiente $\mathrm{N}$ para la síntesis microbiana, se estimó la cantidad de proteína consumida degradable en el rumen (DIP) requerida por los microorganismos, así como el DIP potencial suministrado por la pradera mediante las ecuaciones 5 y 6 .

Cuadro 2. Requerimientos de mantención y ganancia mensual para un novillo Angus en engorda (NRC 2000). Maintenance and gain requirements per month for a finishing Angus steer (NRC 2000).

\begin{tabular}{|c|c|c|c|c|c|}
\hline \multirow{2}{*}{ Requerimientos de mantención } & \multicolumn{5}{|c|}{ Peso vivo promedio para el mes, $\mathrm{kg}$} \\
\hline & 396 & 427 & 458 & 487 & 500 \\
\hline NEm, Mcal/d & 6,84 & 7,23 & 7,62 & 7,98 & 8,14 \\
\hline Proteína microbiana, g/d & 337 & 357 & 376 & 394 & 402 \\
\hline \multicolumn{6}{|l|}{ Requerimientos de crecimiento } \\
\hline GDP, kg/d & \multicolumn{5}{|c|}{$\mathrm{NEg}, \mathrm{Mcal} / \mathrm{d}$} \\
\hline 0,80 & 3,62 & 3,83 & 4,03 & 4,22 & 4,31 \\
\hline 1,00 & 4,75 & 5,02 & 5,29 & 5,54 & 5,65 \\
\hline \multirow[t]{2}{*}{1,25} & 5,90 & 6,24 & 6,58 & 6,89 & 7,03 \\
\hline & \multicolumn{5}{|c|}{ Proteína metabolizable, g/d } \\
\hline 0,80 & 220 & 207 & 195 & 183 & 178 \\
\hline 1,00 & 275 & 258 & 242 & 227 & 221 \\
\hline 1,25 & 328 & 308 & 288 & 269 & 261 \\
\hline
\end{tabular}

$\mathrm{NEm}$ = Energía neta de mantención; NEg = Energía neta de ganancia; PM = Proteína metabolizable; GDP = Ganancia diaria de peso. Valores estimados utilizando las ecuaciones del NRC de carne, 2000. 
DIP requerido $(\mathrm{g} / \mathrm{d})=\mathrm{CMS}$ x TDN x EFm x $1000(\mathrm{~g} / \mathrm{kg})$

(Ec 5)

DIP potencial $(\mathrm{g} / \mathrm{d})=\mathrm{CMS} \times \mathrm{PC}$ x DIP x 1,000 $(\mathrm{g} / \mathrm{kg})$

Las características nutricionales de la pradera de la zona sur de Chile fueron establecidas utilizando como referencia la publicación de Anrique y col (2010), cuyo resumen mensual se presenta en el cuadro 3. Para efectos de esta simulación se asumió que la dieta del animal correspondió en un $100 \%$ a la pradera, no existiendo suplementación ni variaciones que afectasen los valores de PC definidos. El consumo de $\mathrm{N}$ se estimó mediante el cociente PM/6,25, en este caso PM corresponde al valor de proteína metabolizable y 6,25 al contenido promedio de $\mathrm{N}$ de las proteínas. Finalmente, la eficiencia de utilización del $\mathrm{N}$ para cada ecuación evaluada, así como le eficiencia promedio, se determinó mediante la siguiente ecuación:

EUN $(\%)=(($ Consumo $\mathrm{N}$ - Excreción N) / Consumo

$\mathrm{N}) * 100$

\section{RESULTADOS}

El cuadro 2 presenta la evolución mensual de los requerimientos de proteína metabolizable (PM) de mantención y crecimiento del tipo de animal en estudio. Se observa que los requerimientos de PM de mantención aumentan con la edad del animal, mientras que los requerimientos de PM para ganancia disminuyen con el paso del tiempo. En tanto los requerimientos energéticos (energía neta) para mantención y ganancia aumentan con el tiempo. Asimismo, las características nutricionales de la pradera en estudio (cuadro 3) resultan ser adecuadas para satisfacer los requerimientos energéticos y proteicos de la categoría animal establecida, como lo demuestran también los trabajos publicados por Goic (2005) y Goic e Iraira (2005). Los valores promedios de calidad de la pradera para el periodo de estudio corresponden a 20,8\% PC; 1,73 Mcal ENm/kg MS y 1,11 Mcal ENg/kg MS. Apreciándose una disminución en la calidad de la pradera en la medida que se acerca el periodo estival.

La producción de proteína microbiana $\left(\mathrm{P}_{\mathrm{Mic}}\right)$ se presenta en el cuadro 4, observándose un aumento de esta con el paso de los meses. Este aumento en la producción de $\mathrm{P}_{\text {Mic }}$ se debe al mayor consumo de materia seca del animal como resultado del incremento de peso vivo, aun cuando los contenidos de PC disminuyen en el tiempo. Por el contrario, los valores de UIP disminuyen en el tiempo producto del menor contenido de PC de la pradera y la baja proporción de ésta que escapa a la degradación ruminal. Esto último es el resultado de la maduración de las plantas, lo que conlleva una disminución en la calidad de la pradera. En general, se aprecia que la cantidad de PM que suministra la pradera, esto es la suma de UIP $+\mathrm{P}_{\text {Mic }}$, resultan ser superiores a los requerimientos del animal durante todo el periodo de engorda como se puede observar en la figura 1, esto muestra que el balance mensual de PM para el periodo de estudio es positivo. El consumo de $\mathrm{N}$ estimado presenta un patrón similar al de la cantidad de $\mathrm{N}$ excretado.

La figura 2 resume la cantidad estimada de $\mathrm{N}$ consumido y excretado, observándose un rango de excreción de $\mathrm{N}$ que fluctúa entre un 83,0 y 89,2\% (promedio $86,6 \%$ ) cuando se utilizaron las ecuaciones de regresión desarrolladas por Yan y col (2007). El valor promedio de N que se excreta es de 107,7 y 104,9 g/d para las ecuaciones 1 y 2, respectivamente. Esto representa una EUN de 13,37\%. Sin embargo, al utilizar el modelo dinámico de Guo y Zoccarato (2005) se obtienen valores de excreción levemente inferiores, los que fluctúan entre 80,6 y 86,5\% del $\mathrm{N}$ consumido (promedio 83,7\%), observándose un incremento en las excreciones con el transcurso del tiempo. La EUN del modelo de Guo y Zoccarato fue ligeramente superior al alcanzando con las ecuaciones de regresión de Yan y col (2007) 16,3\% vs. 13,4\%, respectivamente.

Cuadro 3. Composición nutritiva mensual de la pradera fertilizada de la zona sur de Chile (Anrique y col 2010). Monthly nutritional composition of fertilized pastures in southern Chile (Anrique y col 2010).

\begin{tabular}{lccccccccccc}
\hline \multicolumn{1}{c}{ MES } & $\begin{array}{c}\text { MS } \\
\%\end{array}$ & $\begin{array}{c}\text { PC } \\
\%\end{array}$ & $\begin{array}{c}\text { FDA } \\
\%\end{array}$ & $\begin{array}{c}\text { FDN } \\
\%\end{array}$ & $\begin{array}{c}\mathrm{EM} \\
\mathrm{Mcal} / \mathrm{kg}\end{array}$ & $\begin{array}{c}\mathrm{ENm} \\
\mathrm{Mcal} / \mathrm{kg}\end{array}$ & $\begin{array}{c}\mathrm{ENg} \\
\mathrm{Mcal} / \mathrm{kg}\end{array}$ & $\begin{array}{c}\mathrm{TDN} \\
\%\end{array}$ & $\begin{array}{c}\mathrm{EE} \\
\%\end{array}$ & $\begin{array}{c}\mathrm{FC} \\
\%\end{array}$ & $\begin{array}{c}\mathrm{Ce} \\
(\%)\end{array}$ \\
\hline Octubre & 14,6 & 28,6 & 38,4 & 21,0 & 2,81 & 1,87 & 1,23 & 83,5 & 3,3 & 21,3 & 9,2 \\
Noviembre & 16,1 & 21,8 & 43,3 & 24,1 & 2,71 & 1,79 & 1,16 & 83,1 & 2,7 & 22,3 & 8,6 \\
Diciembre & 18,2 & 17,9 & 49,7 & 28,7 & 2,62 & 1,71 & 1,09 & 82,2 & 2,4 & 22,8 & 8,4 \\
Enero & 24,0 & 18,4 & 48,7 & 28,4 & 2,57 & 1,66 & 1,05 & 82,2 & 2,6 & 28,7 & 8,5 \\
Febrero & 26,4 & 17,4 & 48,3 & 29,0 & 2,53 & 1,63 & 1,02 & 82,1 & 2,7 & 28,9 & 9,3 \\
\hline
\end{tabular}

MS = Materia Seca; PC = Proteína Curda FDA = Fibra Detergente Ácido; FDN = Fibra Detergente Neutro; EM = Energía Metabolizable; ENm = Energía Neta de mantención; ENg = Energía Neta de ganancia; TDN = Nutrientes Digestibles Totales; EE = Extracto Etéreo; FC = Fibra Cruda; y Ce = Cenizas.

Los valores de UIP y DIP fueron estimados en base a la información publicada por Anrique y col (2010), así para el mes de octubre los valores utilizados fueron de 8,0 y $81,9 \%$ respectivamente, mientras que para el resto de los meses fue de 10,6 y $75,4 \%$ respectivamente. 
Cuadro 4. Resumen de la estimación del balance nutricional y excreción de nitrógeno mensual. Summary of the estimated nutritional balance and excretion of nitrogen per month.

\begin{tabular}{|c|c|c|c|c|c|}
\hline & Octubre & Noviembre & Diciembre & Enero & Febrero \\
\hline Proteína Microbiana, g/d* & 605 & 648 & 688 & 732 & 751 \\
\hline UIP, $g / d^{\gamma}$ & 100 & 82 & 72 & 79 & 77 \\
\hline Proteína Metabolizable, $\mathrm{g} / \mathrm{d}^{\gamma}$ & 705 & 730 & 760 & 810 & 828 \\
\hline DIP requerido, $\mathrm{g} / \mathrm{d}^{\gamma}$ & 945 & 1,013 & 1,076 & 1,143 & 1,174 \\
\hline DIP potencial, $\mathrm{g} / \mathrm{d}^{\gamma}$ & 2,291 & 1,884 & 1,654 & 1,814 & 1,760 \\
\hline Balance DIP & 1,346 & 871 & 578 & 671 & 586 \\
\hline Consumo N, g/d (CN) & 113 & 117 & 122 & 130 & 132 \\
\hline Excreción N, g/d (Ec1) $)^{\psi}$ & 101 & 104 & 108 & 112 & 114 \\
\hline Excreción N, g/d (Ec2) $)^{\Omega}$ & 94 & 99 & 105 & 112 & 115 \\
\hline Eficiencia utilización N (Ec1), \% & 10,8 & 11,0 & 11,5 & 13,5 & 14,0 \\
\hline Eficiencia utilización N (Ec2), \% & 17,0 & 15,8 & 13,9 & 13,5 & 13,1 \\
\hline Eficiencia modelo dinámico, \% & 19,4 & 17,9 & 16,3 & 14,5 & 13,5 \\
\hline
\end{tabular}

* Proteína Metabolizable $=$ UIP $+\mathrm{P}_{\mathrm{Mic}}$

$\gamma$ Valores de proteína by pass (UIP), proteína microbiana $\left(\mathrm{P}_{\mathrm{Mic}}\right)$, proteina degradable en el rumen requerida (DIP requerido) y proteina degradable en el rumen potencial (DIP potencial) estimadas según ecuaciones 3, 4, 5 y 6 respectivamente.

* Ecuación 1: Excreción N, (g/d) $=[0,550+0,032 \times \mathrm{CN} / \mathrm{CMS}] \times \mathrm{PV}^{0,75}+15$ (ver detalles en el texto) .

$\Omega$ Ecuación 2: Excreción $\mathrm{N},(\mathrm{g} / \mathrm{d})=0,609+\mathrm{CN}+0,599 \times \mathrm{PV}^{0,75}-24,7$ (ver detalles en el texto).

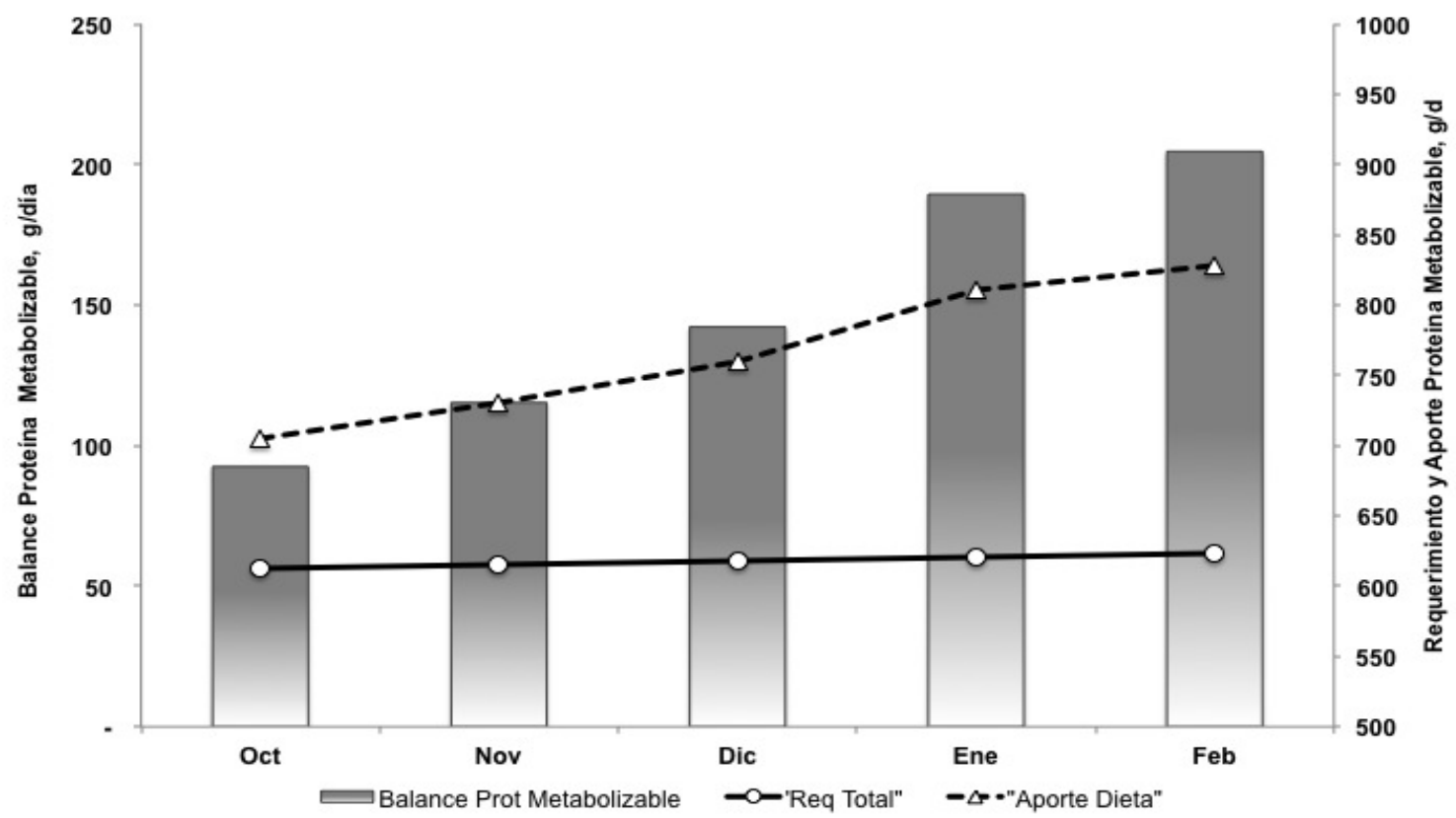

Figura 1. Balance mensual de proteína metabolizable, requerimientos de mantención y ganancia. Monthly metabolizable protein balance, maintenance and gain requirements.

\section{DISCUSIÓN}

En los últimos años, la mayoría de los países del mundo y en forma especial los de la Unión Europea han tratado de reducir el $\mathrm{N}$ excretado, debido a la percepción generalizada del $\mathrm{N}$ como un contaminante frecuente, $\mathrm{y}$ por su potencial daño al medioambiente (Goodale y col
2011, Guo y Zoccarato 2005). Galloway y col (2008) plantearon los efectos negativos que el $\mathrm{N}$ tiene sobre el medioambiente, particularmente por el creciente uso de compuestos nitrogenados que son utilizados como insumo para promover un aumento en la producción de alimentos. En efecto, los mismo autores agregan que la creación de $\mathrm{Nr}$ se ha incrementado en 120\% desde 1970. 


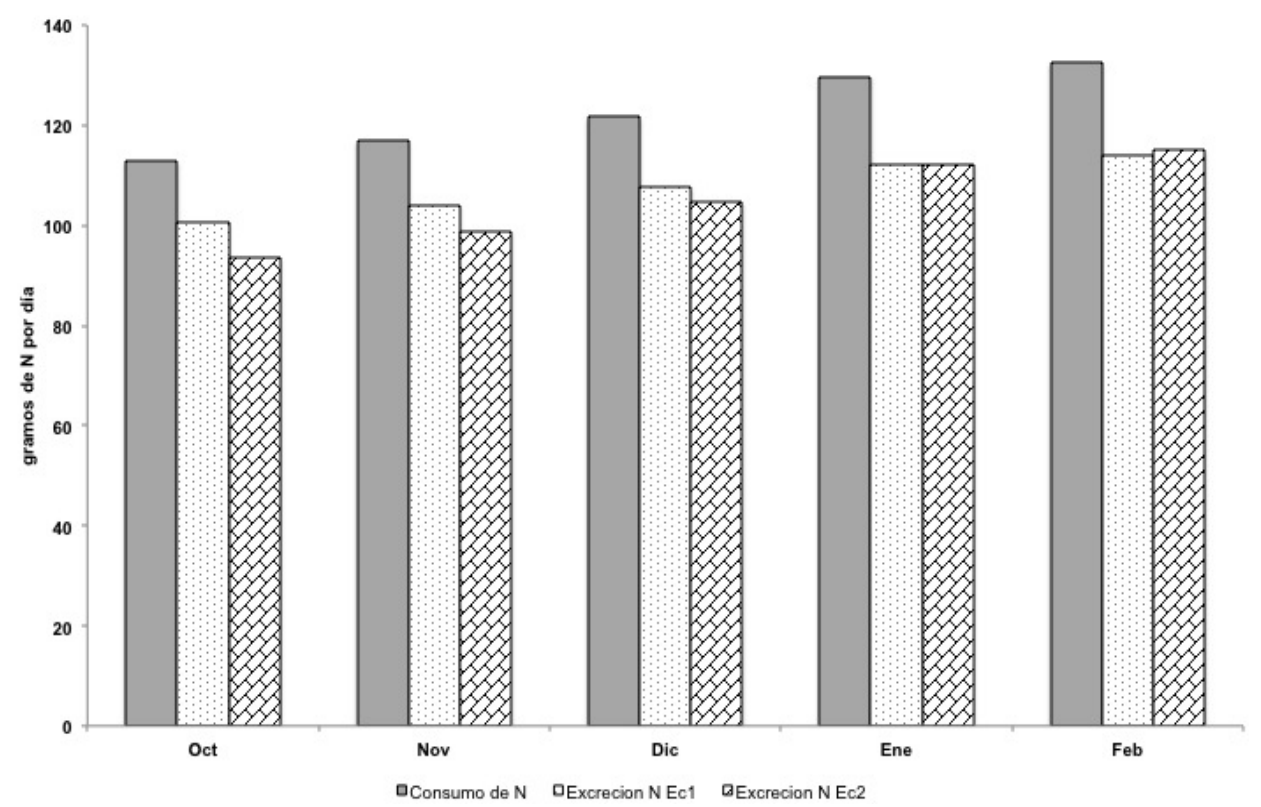

Figura 2. Estimación del nitrógeno consumido y excretado por día.

$\mathrm{Ec} 1=[0,550+0,032 \times \mathrm{CN} / \mathrm{CMS}] \times \mathrm{PV}^{0,75}+15 ; \mathrm{Ec} 2=0,609+\mathrm{CN}+0,599 \times \mathrm{PV}^{0,75}-24,7 ;$ donde $\mathrm{CN}=$ consumo nitrógeno; $\mathrm{CMS}=$ Consumo materia seca y PV = Peso vivo (Yan y col 2007).

Estimation of daily intake and excretion of nitrogen.

$\mathrm{Ec} 1=[0,550+0,032 \times \mathrm{CN} / \mathrm{CMS}] \times \mathrm{PV}^{0,75}+15 ; \mathrm{Ec} 2=0,609+\mathrm{CN}+0,599 \times \mathrm{PV}^{0,75}-24,7$; where $\mathrm{CN}=$ nitrogen intake; CMS = Dry matter intake and $\mathrm{PV}=$ Body weight (Yan y col 2007).

En el caso de Chile, según datos de ODEPA (2011), las importaciones de fertilizantes y abonos químicos (nitrógeno, fósforo y potasio) crecieron significativamente en los últimos 20 años desde 95 ton/año en 1990 a 10.824 ton/año en el 2010. De forma similar, otros reportes como el de Chatzimpiros y Barles (2010) reflejan un creciente uso de $\mathrm{N}$ en la producción animal. Los mismos autores estiman que el suministro $\mathrm{N}$ para sustentar la producción de forraje necesaria para la producción de carne y leche en el oeste de Francia fue de alrededor de $50 \mathrm{~kg}$ N/ha a mediados del siglo 19; $54 \mathrm{~kg} \mathrm{~N} / \mathrm{ha}$ a comienzos del siglo 20 y $150 \mathrm{~kg} \mathrm{~N} / \mathrm{ha}$ en el siglo 21 . En Chile, existen limitados estudios respecto de la EUN en la producción agropecuaria, y son inexistentes en la producción bovina de carne. No obstante, las consideraciones medioambientales están recibiendo cada vez más atención en las agendas políticas, sociales y económicas de diversos países del mundo. En este sentido, la utilización de modelos para reducir al mínimo la excreción de $\mathrm{N}$ se hace cada vez más necesaria. Guo y Zoccarato (2005), plantean que su modelo puede ser utilizado por gobiernos y agricultores para organizar las actividades de producción animal, manteniendo así el equilibrio económico y las prácticas ambientales sostenibles. En efecto, se espera que las exigencias en la producción de alimentos de origen animal de alta calidad reduzcan al mínimo los efectos negativos sobre el medioambiente y en lo posible aumenten la eficiencia productiva.
El aumento de la intensificación en la ganadería ha sido asociado con mayores daños ambientales. Este deterioro es ocasionado, en parte, por las excreciones generadas por los animales que incluyen gases, orina y heces, las que en conjunto contribuyen al efecto invernadero, la contaminación del suelo y, especialmente de las aguas superficiales y subterráneas (Ibarra y col 2006). A modo de ejemplo, De Klein y col (2009) indican que la principal fuente de $\mathrm{N}_{2} \mathrm{O}$ en los sistemas de pastoreo son las excretas depositadas por animales o las excretas aplicadas a la tierra como abono, las que comúnmente provienen de las salas de ordeño o de los sistemas de alojamiento invernal (galpones de engorda). Si se considera que cada animal excreta alrededor de 2,2 kg/animal/d (Erickson y col 1999) y que la masa ganadera en Chile alcanza cerca de 3,7 millones de cabezas (según datos del último CEN$\mathrm{SO}$ ), se estarían liberando diariamente cerca de 10.340 ton de estiércol o el equivalente a 55,94 ton/d de N. Lo anterior no sólo genera pérdidas económicas, sino que también un deterioro ambiental (Alfaro y Salazar 2005). Por otro lado Capper (2011), comparó los sistemas de producción de carne bovina de 1977 vs. 2007, concluyendo que los sistemas intensivos de producción de carne requieren considerablemente menos recursos para lograr el mismo nivel de producción $(69,9 \%$ menos animales; 18,6\% menos de alimentos; $12,19 \%$ menos de agua; y solo un $67 \%$ de la tierra), reduciendo la huella de carbono en un $16,3 \%$. Asimismo, la cantidad de desechos por 
producto representan una reducción de $18,1 \% ; 17,7 \%$ y $12,0 \%$ de estiércol, $\mathrm{CH}_{4}$ y $\mathrm{N}_{2} \mathrm{O}$, respectivamente.

En términos de modelación se puede señalar que la excreción de $\mathrm{N}$ es igual a la ingesta de $\mathrm{N}$ menos el $\mathrm{N}$ retenido en el cuerpo y a su vez el $\mathrm{N}$ retenido representa los requerimientos del animal (Guo y Zoccarato 2005). Sin embargo, los requerimientos de PM establecidos en el NRC para bovinos de carne se basan en el método factorial, el que incluye las pérdidas de la fracción metabólica fecal, orina, piel, crecimiento, crecimiento fetal y leche. Las pérdidas metabólicas fecales, orina y piel representan los requerimientos necesario para el mantenimiento (NRC 2000). En la presente simulación se observan cambios en los requerimientos de proteína metabolizable de mantención, los que se explicarían por el incremento de la masa muscular al inicio del periodo de engorda y por la menor tasa de deposición de músculo al acercarse a la edad de faena (Vasconcelos y col 2007). No obstante, cuando se compara el contenido promedio de PC las dietas de engorda en USA, que alcanza al 13\% (Castro Bulle y col 2007, Erickson y Klopfenstein 2010, Galyean 1996), con los contenidos de la pradera en estudio, se observa que esta última suministra más PC y en consecuencia más PM de la que el animal necesita. Esto es particularmente importante durante la primavera, en donde la oferta alcanza 2,2 veces el equivalente a la necesidad de PC del animal en engorda. Klopfenstein y col (2002) señalan que un exceso de proteína en la dieta no tiene valor para los animales, siendo éste excretado principalmente en la orina como Urea-N. Lo anterior no sólo tiene importantes repercusiones económicas y productivas, dado que se considera la proteína como el nutriente más costoso, sino que también por el consecuente daño ambiental que ocasiona (Cole y col 2006). En este mismo sentido, Salazar (2005) agrega que una dieta balanceada maximiza el uso de los nutrientes por los animales y reduce las pérdidas en heces y orina. Sin embargo, lograr balancear la dieta en sistemas pastoriles es difícil, dada la multiplicidad de factores que actúan en forma conjunta.

El concepto de proteína metabolizable adoptado por el NRC a partir de 1996, separa las demandas del animal de aquellas de los microorganismos del rumen (NRC 2000). Así entonces, el $\mathrm{N}$ que suministra la dieta que un animal consume es aprovechado en primer lugar por los microbios ruminales para transformarlo en $\mathrm{P}_{\text {Mic }} \mathrm{y}$, posteriormente, por el animal que aprovecha la proteína verdadera que escapa a la degradación ruminal y la $\mathrm{P}_{\text {Mic }}$, los que llegan al intestino delgado, lugar donde son digeridos y absorbidos (Erickson y col 1998, Patterson y col 2006, Vasconcelos y col 2007). En general, la mayoría de los microbios del rumen utilizan $\mathrm{NH}_{3}-\mathrm{N}$ como fuente primaria de $\mathrm{N}$, mientras que algunas especies requieren de otros compuestos nitrogenados para un crecimiento más rápido y eficiente (Owens y Bergen 1983). Lo anterior se explicaría por la hidrólisis de urea reciclada en el rumen.

Los valores de DIP requeridos representan la cantidad de proteína que debe estar disponible para satisfacer la síntesis de $\mathrm{P}_{\text {Mic }}$ en el rumen. En tanto, el DIP potencial es un indicador de la cantidad de DIP que podría estar disponible para los microorganismos ruminales dadas las características del alimento, en este caso la pradera en estudio. Un balance positivo del DIP implica un exceso de $\mathrm{N}$ en la dieta, el que será finalmente reciclado o eliminado del sistema. Por el contrario, un balance negativo implica la necesidad de suplementar la dieta con $\mathrm{N}$ para maximizar la síntesis de $\mathrm{P}_{\text {Mic }}$. No obstante lo anterior, existe evidencia de que dietas con un leve balance negativo de DIP no tienen un efecto detrimental sobre el desempeño productivo, mientras que sí reducen las pérdidas de N (Huhtanen y col 2008, Valkeners y col 2008). Esto se explicaría por la capacidad de los animales de reciclar el $\mathrm{N}$ a través de la saliva. Es importante señalar que el interés en la PM se debe a que ésta representa la alternativa más económica de suministrar aminoácidos al animal. En este mismo sentido Owens y Bergen (1983) señalan que la dinámica del $\mathrm{NH}_{4}$ ruminal $\mathrm{y}$, en consecuencia el crecimiento microbiano involucra distintas fuentes y destinos del N, destacando como fuentes: 1) la degradación de la proteína verdadera y NNP; 2) hidrólisis de la urea reciclada en el rumen y 3) degradación de protoplasma microbial. Asimismo, los destinos incluyen: 1) uso por los microbios; 2) absorción a través de la pared ruminal y 3) avance hacia el omaso. Así, entonces, cuando el N captado por los microorganismos del rumen es menor que el liberado en el rumen, la concentración de $\mathrm{NH}_{3}$ en el rumen aumenta, siendo absorbido a través de la pared ruminal y transportado al hígado (Pulido 2010).

Los valores estimados de $\mathrm{N}$ excretado en el presente estudio concuerdan con lo indicado por Klopfenstein y col (2002), quienes señalan que la eficiencia de convertir proteína dietética en músculo en el ganado de carne es una de las más bajas, siendo inferior al 20\%. En otro estudio realizado en Francia, considerando tanto la producción lechera como de carne, se plantea que el potencial de eficiencia en la conversión de los vegetales en proteínas animales ha sido más que duplicado en el último siglo, pasando de menos del $9 \%$ en el siglo 19 a cerca de $20 \%$ en el siglo 21 (Chatzimpiros y Barles 2010). Los valores de la presente simulación se encuentran bajo el $20 \%$ señalado por estos autores.

Una vía importante para la mitigación en la excreción de $\mathrm{N}$ es el aumento en la EUN por parte de los animales. En este sentido, cobra relevancia el concepto de selección de animales en base al consumo residual de alimento (Residual Feed Intake = RFI). Éste representa una medida de eficiencia de los animales y es independiente del nivel de producción, del tamaño y crecimiento del ganado de carne (Herd y Arthur 2009). Los mismos autores indican que las proporciones en variación de RFI son explicadas en un $37 \%$ por la síntesis de proteína, lo que refleja una importante variación entre animales. Una segunda opción es ajustar la dieta de los animales a sus requerimientos en las distintas etapas productivas, ya 
sea mediante el sistema de PC o bien proteína matebolizable. Este tipo de práctica de alimentación mejorada comprende la agrupación de los animales por género y por fase de producción, así como también lograr un aumento en los índices de conversión de alimento mediante la preparación de raciones en función de las necesidades fisiológicas del animal. Sin embargo, incluso cuando se implementan buenas prácticas de manejo para minimizar la excreción de $\mathrm{N}$, grandes cantidades continúan siendo excretadas (Steinfield y col 2006). Rotz (2004) plantea que la reducción en la pérdida de $\mathrm{N}$ en las explotaciones ganaderas debe basarse en un manejo integral de los rebaños y de la alimentación de éstos. Un claro ejemplo de la utilización del sistema de ajuste de la dieta a los requerimientos del ganado fue validado por Erickson y col (1999), quienes sugieren que la alimentación por etapas o fases resulta en menores emisiones de N. En su estudio, estos autores compararon el efecto de una dieta convencional rica en concentrados $(92,5 \%)$ con un 13,5 $\%$ PC vs. una dieta por etapas que ajustaba el suministro de $\mathrm{N}$ a los requerimientos de los animales según la fase de engorda (utilizando el sistema de PM), pero manteniendo el mismo nivel de concentrados. Ellos lograron reducir las excreciones de $\mathrm{N}$ en un $22 \%$, mientras que el $\mathrm{N}$ volatilizado se redujo en un $32 \%$. Los mismos autores reportaron reducciones de $\mathrm{N}$ excretado de un $13 \%$ y un $15 \%$ de $\mathrm{N}$ volatilizado, utilizando terneros durante la época invernal/primaveral. Las diferencias en volatilización podrían explicarse por las temperaturas más frías en este último estudio. Sin embargo, las menores pérdidas están más bien asociadas a menores ingestas de $\mathrm{N}$ y a una menor cantidad de $\mathrm{N}$ perdido en la orina, lo que resulta en una menor volatilización del N (Erickson y Klopfenstein 2010). Keim y Anrique (2011), en una revisión respecto del tema, plantean una serie de alternativas que han sido evaluadas para mejorar la EUN, principalmente a través de un efecto de dilución mediante diversas estrategias, entre ellas es posible mencionar: a) reducción del consumo de $\mathrm{N}$, en base a la selección de especies forrajeras con menor contenido de $\mathrm{N}$ o bien mediante la reducción de la fertilización nitrogenada entre pastoreos. Sin embargo, estas alternativas pueden resultar en una menor producción y/o afectar la calidad nutricional; b) Sincronización del suministro de carbohidratos y $\mathrm{N}$ a nivel ruminal; c) Incremento del relación contenido de carbohidratos solubles/proteína cruda (WSC/PC) en las especies forrajeras; d) utilización de variedades forrajeras ricas en azúcares.

La utilización del sistema de PM permite formular dietas de engorda con más precisión que con el sistema de PC, de manera tal de satisfacer los requerimientos del animal sin caer en el exceso en el suministro de proteína. El reto de este sistema no es sólo satisfacer los requerimientos globales del animal, sino que el de los microorganismos ruminales, de manera tal de evitar una sobrealimentación y la consecuente pérdida de N. Esto es especialmente difícil de implementar con precisión, particularmente bajo condiciones como las planteadas en este estudio (sistema a pastoreo) debido a lo variable en la calidad del alimento, selección realizada por los animales y los cambios en degradabilidad de la proteína a nivel ruminal que ello conlleva.

En Chile, a la fecha, no existen estudios que cuantifiquen la excreción de $\mathrm{N}$ de animales provenientes de engorda en base a praderas o en confinamiento. Por ello, los resultados de la presente simulación representan un primer esfuerzo por determinar la EUN en ganadería y el potencial efecto contaminante del $\mathrm{N}$ excretado en estos sistemas productivos. Cabe señalar que las altas cargas de fertilizante nitrogenado aplicados tanto a praderas como a cultivos, además de la baja EUN en los sistemas ganaderos representan una alta carga de $\mathrm{N}$ al ecosistema $\mathrm{y}$, al mismo tiempo, generan una demanda creciente por estudios a nivel predial que permitan cuantificar el impacto del $\mathrm{N}$ y otros minerales en la sustentabilidad de los sistemas productivos del sur de Chile. Si a este escenario se suman las proyecciones internacionales de mayor demanda de carne bovina, mayores exigencias ambientales y de bienestar animal, resulta evidente la necesidad de ofrecer a los productores ganaderos estrategias de mitigación validadas localmente, las que por un lado permitan mejorar su eficiencia productiva y al mismo tiempo aseguren la sustentabilidad económica y ambiental del negocio.

En el caso particular de los sistemas pastoriles, los resultados de esta simulación indican una muy baja EUN en las engordas. Esto plantea el desafío de desarrollar líneas de investigación, desarrollo y validación de tecnologías y estrategias de mitigación que permitan reducir las excreciones de $\mathrm{N}$ y de otros minerales al ecosistema. Entre ellas, sin duda la primera alternativa es ajustar la oferta de nutrientes a los requerimientos del animal según su etapa productiva (balancear las raciones). Otras alternativas a considerar incluyen la implementación del sistema de PM, sincronización energía:proteína en el rumen y dilución del $\mathrm{N}$ ingerido en la dieta a través de diversos manejos alimenticios. Un primer esfuerzo debiera centrarse en la determinación del nivel y evolución de la degradabilidad de la proteína, digestibilidad de la materia orgánica y el establecimiento de la relación WSC/PC de las praderas del sur de Chile, a través del año. Así, entonces, la generación de este tipo de información es clave para la implementación de dichas estrategias.

\section{REFERENCIAS}

Alfaro M, F Salazar. 2005. Ganadería y contaminación difusa, implicancias para el sur de Chile. Agric Tec 65, 330-340.

Anrique R, R Fuchslocher, S Iraira, R Saldaña. 2010. Composición de alimentos para el ganado bovino. $4^{\mathrm{a}} \mathrm{ed}$. Consorcio Lechero, Universidad Austral de Chile, Valdivia, Chile.

Balocchi O. 2011. La pradera como alimento. En: Pulido R, Parga J, Lanuza F, Balocchi O (eds). Suplementación de 
vacas lecheras a pastoreo. Osorno, Chile, Pp 11-20

Capone DG, R Popa, B Flood, KH Nealson. 2006. Follow the Nitrogen. Science 312, 708-709.

Capper JL. 2011. The environmental impact of beef production in the United States: 1977 compared with 2007. J Anim Sci 89, 4249-4261.

Castro Bulle FCP, PV Paulino, AC Sanches, RD Sainz. 2007. Growth, carcass quality, and protein and energy metabolism in beef cattle with different growth potentials and residual feed intakes. J Anim Sci 85, 928-936.

Chatzimpiros P, S Barles. 2010. Nitrogen, land and water inputs in changing cattle farming systems. A historical comparison for France, $19^{\text {th }}-21^{\text {st }}$ centuries. Sci Total Environ 408, 4644-4653.

Cole NA, PJ Defoor, ML Galyean, GC Duff, JF Gleghorn. 2006. Effects of phase-feeding of crude protein on performance, carcass characteristics, serum urea nitrogen concentrations, and manure nitrogen of finishing beef steers. J Anim Sci 84, 3421-3432.

De Klein CAM, C Pinares-Patino, GC Waghorn. 2009. Greenhouse gas emmisions. In: McDowell RW (ed). Environmental impacts of pasture-based farming. CAB International, Oxford, UK, Pp 1-32.

Dumont J, F Salazar. 2001. Manejo y utilización de purines en planteles ganaderos. En: Opazo L, Torres A, Siebald E (eds). Hacia un nuevo estilo productivo. Centro Nacional de Investigaciones INIA. Serie Actas $N^{\circ}$ 9, Remehue, Osorno, Chile, Pp 29-33.

Elizondo J. 2002. Estimación lineal de los requerimientos nutricionales del NRC para ganado de leche. Agron Mesoam 13, 41-44.

Erickson GE, T Milton, TJ Klopfenstein. 1998. Evaluation of 1996 NRC for protein and phosphurus requirements of finishing cattle. Nebraska Beef Report MP69-A. University of Nebraska, Lincoln Nebraska, Nebraska, USA, Pp 84-85.

Erickson GE, TJ Klopfenstein, T Milton, D Herold. 1999. Effects of matching protein requirements on performance and waste management in the feedlot. Nebraska Beef Report MP71. University of Nebraska, Lincoln Nebraska, Nebraska, USA, Pp 60-63.

Erickson GE, TJ Klopfenstein. 2010. Nutritional and management methods to decrease nitrogen losses from beef feedlots. J Anim Sci 88, E172-180.

Francis CA, JM Beman, MMM Kuypers. 2007. MINI-REVIEW New processes and players in the nitrogen cycle: the microbial ecology of anaerobic and archaeal ammonia oxidation. ISME J 1, 19-27.

Galloway JN, FJ Dentener, DG Capone, EW Boyer, RW Howarth, SP Seitzinger, GP Asner, CC Cleveland, PA Green, EA Holland, DM Karl, AF Michaels, JH Porter, AR Townsend, CJ Vorosmarty. 2004 Nitrogen cycles: past, present, and future. Biogeochemistry 70, 153-226.

Galloway JN, AR Townsend, JW Erisman, M Bekunda, ZC Cai, JR Freney, LA Martinelli, SP Seitzinger, MA Sutton. 2008. Transformation of the nitrogen cycle: recent trends, questions, and potential solutions. Science 320, 889-892.

Galyean ML. 1996. Protein levels in beef cattle finishing diets: Industry application, university research, and systems results. J Anim Sci 74, 2860-2870.

Galyean ML. 2000. Environmental stewardship in the future:
Nutrient management issues and options for beef cattle feeding operations. J Anim Sci 79, 1-9.

Goodale CL, NB Dise, MA Sutton. 2011. Introduction. Special issue on nitrogen deposition, critical loads, and biodiversity. Environ Pollut 159, 2211-2213.

Guo K, I Zoccarato. 2005. A dynamic model to predict the nitrogen excretion in growing-finishing cattle. Ecol Model 187, 219-231.

Herd RM, PF Arthur. 2009. Physiological basis for residual feed intake. J Anim Sci 87, E64-71.

Huhtanen P, JI Nousiainen, M Rinne, K Kytölä, H Khalili. 2008. Utilization and partition of dietary nitrogen in dairy cows fed grass silage-based diets. J Dairy Sci 91, 3589-3599.

Ibarra D, L Latrille, F Wittwer. 2006. Incremento en la proteína no degradable en el rumen de vacas lecheras 2. Efectos sobre utilización y excreción de nitrógeno. Arch Med Vet 38 219-225.

Keim JP, R Anrique. 2011. Nutritional strategies to improve nitrogen use efficiency by grazing dairy cows. Chil J Agr Res 71, 623-633.

Klopfenstein TJ, R Angel, GL Cromwell, GE Erickson, DG Fox, C Parsons, LD Satter, AL Suttton. 2002. Animal diet modification to decrease the potential for nitrogen and phosphorus pollution Council for Agricultural Science and Technology (CAST) No. 21, Pp 16.

NRC, National Research Council. 2000. Nutrient requirement of beef cattle, update 2000. National Academy Press, Washington DC, USA.

Oaks A. 1994. Efficiency of nitrogen utilization in C3 and C4 cereals. Plant Physiol 106, 407-414.

Owens FN, WG Bergen. 1983. Nitrogen metabolism of ruminant animals: Historical perspective, current understanding and future implications. J Anim Sci 57, 498-518.

Patterson HH, DC Adams, TJ Klopfenstein, GP Lardy. 2006. Application of the 1996 NRC to protein and energy nutrition of range cattle. Prof Anim Sci 22, 307-317.

Pulido R. 2010. Dinámica del nitrógeno amoniacal y pH ruminales en vacas a pastoreo. En: Contreras P, Noro M (eds). Rumen: Morfosiología, trastornos y modulación de la actividad fermentativa. Consorcio Lechero - Universidad Austral de Chile, Valdivia, Chile, Pp 61-68.

Pulido RG, R Muñoz, C Jara, OA Balocchi, JP Smulders, F Wittwer, P Orellana, M O'Donovan. 2010. The effect of pasture allowance and concentrate supplementation type on milk production performance and dry matter intake of autumn-calving dairy cows in early lactation. Livest Sci 132, 119-125.

Rotz CA. 2004. Management to reduce nitrogen losses in animal production. J Anim Sci 82, E119-137.

Salazar F. 2005. Utilización y pérdidas de nitrógeno en aplicación de purines y estiércol de lechería Revista Tierra Adentro enero-febrero, 23-25.

Steinfield H, P Gerber, T Wassenaar, V Castel, M Rosales, C De Haan. 2006. La larga sombra del Ganado. FAO, Roma, Italia.

Valkeners D, A Thewis, M Van Laere, Y Beckers. 2008. Effect of rumen-degradable protein balance deficit on voluntary intake, microbial protein synthesis, and nitrogen metabolism in growing double-muscled Belgian Blue bulls fed corn silage-based diet. J Anim Sci 86, 680-690.

Van der Hoek KW. 1998. Nitrogen efficiency in global animal 
production. Environ Pollut 102 (Suppl. 1), 127-132.

Van Horn HH, GL Newton, WE Kunkle. 1996. Ruminant nutrition from an environmental perspective: factors affecting whole-farm nutrient balance. J Anim Sci 74, 3082-3102.

Vasconcelos JT, LO Tedeschi, DG Fox, LW Greene. 2007. Review: Feeding nitrogen and phosphorus in beef cattle feedlot production to mitigate environmental impacts. Prof Anim Sci 23, 8-17.
Weiss WP, HR Conrad, NR St. Pierre. 1992. A theoreticallybased model for predicting total digestible nutrient values of forages and concentrates. Anim Feed Sci Technol 39, 95-110.

Yan T, JP Frost, TWJ Keady, RE Agnew, CS Mayne. 2007. Prediction of nitrogen excretion in feces and urine of beef cattle offered diets containing grass silage. J Anim Sci 85, 1982-1989. 